\title{
C-10
}

\section{COMPORTAMIENTO DE 13 MODELOS DE CINTA DE RIEGO EN CONDICIONES DE INVERNADERO CON AGUA REGENERADA}

\author{
Baeza Cano, R. (1), Zapata Sierra, A.J. (2), Alonso López, F. (1), Fernández Guerrero, A.J.
}

(2), Contreras París, J.I. (1)

\author{
${ }^{1}$ Instituto de Investigación y Formación Agraria y Pesquera de Andalucía (IFAPA), Centro La \\ Mojonera, Camino San Nicolás, n¹, 04745 La Mojonera, Almería. \\ rafaelj.baeza@juntadeandalucia.es \\ ${ }^{2}$ Universidad de Almería, Ctra. De Sacramento s/n, 04120 Almería. \\ ajzapata@ual.es
}

\section{Resumen}

El objetivo de este trabajo ha sido seleccionar modelos de cinta de riego con una mejor adaptación a las condiciones de invernadero y riego con aguas regeneradas. Se ha evaluado el comportamiento de 13 modelos comerciales de cintas de riego seleccionados de entre los más utilizados en los cultivos hortícolas al aire libre, y aquellos que, las empresas instaladoras y fabricantes consideren que, por sus características, pueden tener un buen comportamiento con este tipo de aguas.

En la evaluación de campo todos los emisores han presentado valores de uniformidad elevados al comienzo de la campaña de riego. Cinco de las cintas se clasifican como excelentes y el resto como buenas. Al finalizar las 114 horas de trabajo decae sustancialmente la uniformidad. El comportamiento tras la finalización de la primera campaña de riego se puede considerar equiparable al obtenido en un estudio previo con una selección de 20 emisores en ramal convencional y este tipo de aguas.

Se puede concluir que las cintas de riego localizado pueden ser una alternativa a los ramales de riego convencionales cuando se riega con aguas residuales urbanas regeneradas, siempre que se elija un material de una mínima calidad y el ratio de sustitución garantice una uniformidad alta.

\section{1- Introducción y Objetivos}

La situación de déficit hídrico en Andalucía es especialmente crítica en las zonas costeras, especialmente en las Mediterráneas. En la provincia de Almería, donde existe la mayor concentración de cultivos hortícolas bajo invernadero, el déficit estructural es superior a los $100 \mathrm{Hm}^{3}$ (Junta de Andalucía, 2014). La situación de déficit ha obligado a la búsqueda y empleo de nuevas fuentes de agua, principalmente desaladas y residuales urbanas regeneradas (Agencia Andaluza del Agua, 2009; Baeza et al. 2015). El empleo de estas últimas no está exento de problemas, en concreto suelen ser frecuentes los fenómenos de obturación de emisores de riego localizado como consecuencia, principalmente, de la elevada carga microbiológica presente en estas aguas. EI IFAPA ha acometido diversos trabajos previos en los que se ha podido corroborar la problemática en emisores de goteo en ramal de riego convencional, en las que se producen rápidos descensos en la uniformidad de distribución de riego (Baeza et al. 2010, 2012, 2015). Como alternativa se plantea el empleo de cintas de riego de bajo coste que se puedan sustituir antes de perder la uniformidad. El objetivo de este trabajo ha sido seleccionar modelos de cinta de riego con una mejor adaptación a las condiciones de invernadero y riego con aguas regeneradas. Se ha evaluado el comportamiento de 13 modelos comerciales de cintas de riego seleccionados 
de entre los más utilizados en los cultivos hortícolas al aire libre, y aquellos que, las empresas instaladoras y fabricantes consideren que, por sus características, pueden tener un buen comportamiento con este tipo de aguas.

\section{2- Material y Métodos}

En la tabla 1 se muestran las características técnicas de los 13 modelos evaluados. Se han elegido los modelos de cinta de riego, seleccionando los mas empleados en los cultivos de fresa de Huelva y en los cultivos hortícolas de hoja de Andalucía Oriental (en ambas zonas es común el empleo de cintas de riego). Se ha completado la colección para el ensayo consultando a 5 empresas fabricantes que han recomendado varios modelos que consideran, que por sus características, pueden tener un buen comportamiento con este tipo de aguas.

Tabla 1. Modelos de cinta evaluados

\begin{tabular}{|c|c|c|c|c|c|}
\hline $\mathbf{N}^{\circ}$ & Modelo & Fabricante & $\begin{array}{l}\text { Caudal*/Separación } \\
\text { emisores }\left(\mathrm{I} \mathrm{h}^{-1} / \mathrm{cm}\right)\end{array}$ & $\begin{array}{l}\text { Tipología } \\
\text { emisor }\end{array}$ & $\begin{array}{l}\text { Compensación } \\
\text { Caudal }\end{array}$ \\
\hline 1 & $\begin{array}{l}\text { URA-T } \\
\text { Pathfinder }\end{array}$ & CHAMSA & $1,24 / 20$ & Doble pared & NO \\
\hline 2 & $\begin{array}{l}\text { Streamline } \\
16060\end{array}$ & NETAFIN & $0,78 / 20$ & Integrado pastilla & NO \\
\hline 3 & T-Tape & RIVULIS & $1,42 / 20$ & Doble pared & NO \\
\hline 4 & Azudline & AZUD & $1,45 / 30$ & Integrado pastilla & NO \\
\hline 5 & Azudline & AZUD & $2,20 / 20$ & Integrado pastilla & NO \\
\hline 6 & Tal-Drip & $\begin{array}{l}\text { NAAN DAN } \\
\text { JAIN }\end{array}$ & $1,60 / 30$ & Integrado pastilla & NO \\
\hline 7 & Top-Drip & $\begin{array}{l}\text { NAAN DAN } \\
\text { JAIN }\end{array}$ & $1,74 / 40$ & Integrado pastilla & SI \\
\hline 8 & $\begin{array}{l}\text { Streamline } \\
16080\end{array}$ & NETAFIN & $0,69 / 20$ & Integrado pastilla & NO \\
\hline 9 & Rodrip & RIVULIS & $1,67 / 20$ & Doble pared & NO \\
\hline 10 & $\begin{array}{l}\text { URA-T } \\
\text { Pathfinder }\end{array}$ & CHAMSA & $1,25 / 20$ & Doble pared & NO \\
\hline 11 & $\begin{array}{l}\text { Streamline } \\
16080\end{array}$ & NETAFIN & $1,05 / 30$ & Integrado pastilla & NO \\
\hline 12 & Lin One & CAUDAL & $1 / 20$ & Integrado pastilla & NO \\
\hline 13 & P1/Ultra & IRRITEC & $1,1 / 30$ & Integrado pastilla & NO \\
\hline
\end{tabular}

En un banco de ensayo homologado de emisores de riego se ha determinado el coeficiente de variación (ecuación 1) y el rendimiento hidráulico del emisor de cada una de las cintas mediante la función empírica que relaciona el caudal emitido por el emisor en función de la presión de funcionamiento (curva de gasto del emisor) (Keller y Karmeli, 1974) (ecuación 2):

$$
\mathrm{CV}=\mathrm{S} / \mathrm{qm}
$$


Donde:

$\mathbf{S}$ es la desviación típica respecto a la media de los caudales muestreados. qm es el valor medio de los caudales muestreados.

$\mathbf{Q}=\mathbf{k} \mathbf{P}^{\mathbf{x}}$ (Ecuación 2)

Donde:

Q es el caudal de descarga del emisor $\left(\mathrm{L} \mathrm{h}^{-1}\right)$

$\mathbf{k}$ es la constante del emisor

$\mathbf{P}$ presión de trabajo (bar)

$\mathbf{x}$ es el exponente de descarga

El banco de ensayo utilizado se diseñó según la norma UNE 68-075-86 (figura 1), evaluando veinticinco emisores por modelo.

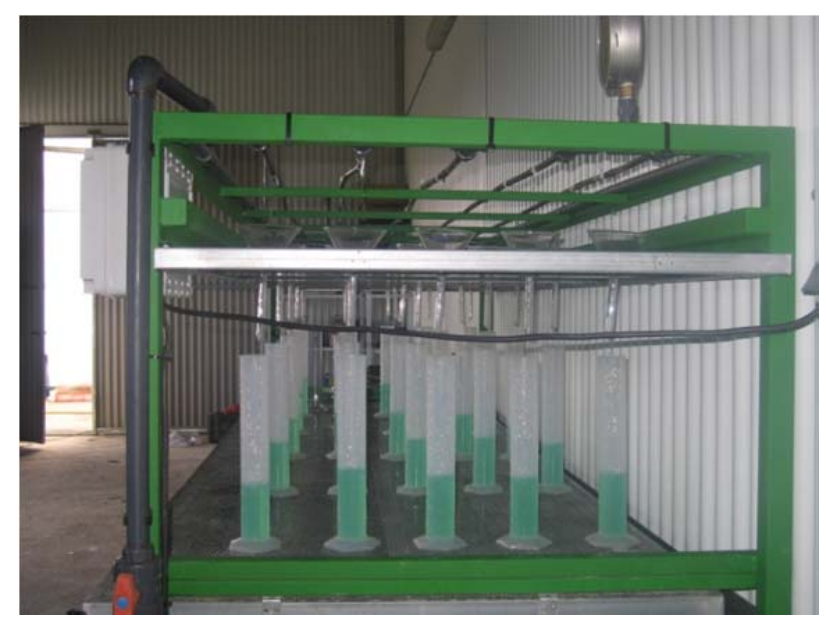

Figura 1. Banco de ensayo diseñado según norma UNE 68-075-86

A continuación en un invernadero experimental multitúnel de $1.200 \mathrm{~m}^{2}$ situado en las instalaciones que posee el Centro IFAPA La Mojonera en La Cañada (Almería) y equipado con control automático de clima y autómata de riego se ha instalado el banco de pruebas en campo de cintas de riego. Haciendo uso del controlador automático de clima se han mantenido unos parámetros climáticos equivalentes a los medidos en un invernadero convencional cultivado con tomate.

Se ha establecido un dispositivo experimental de bloques completos al azar, con tres repeticiones, donde la unidad experimental básica ha sido el lateral de riego (figura 2). Las cintas han sido sometidas a 228 pulsos de riego de 30 minutos de duración y 0,1 MPa de presión. Para cintas con un caudal de $5 \mathrm{I} \mathrm{m}^{-1}$, instaladas a un marco de $1 \mathrm{~m}$, el volumen aplicado equivaldría a $570 \mathrm{I} \mathrm{m}^{-2}$, que corresponde de manera aproximada al consumo de un ciclo largo de tomate (Fernández et al. 2001). 


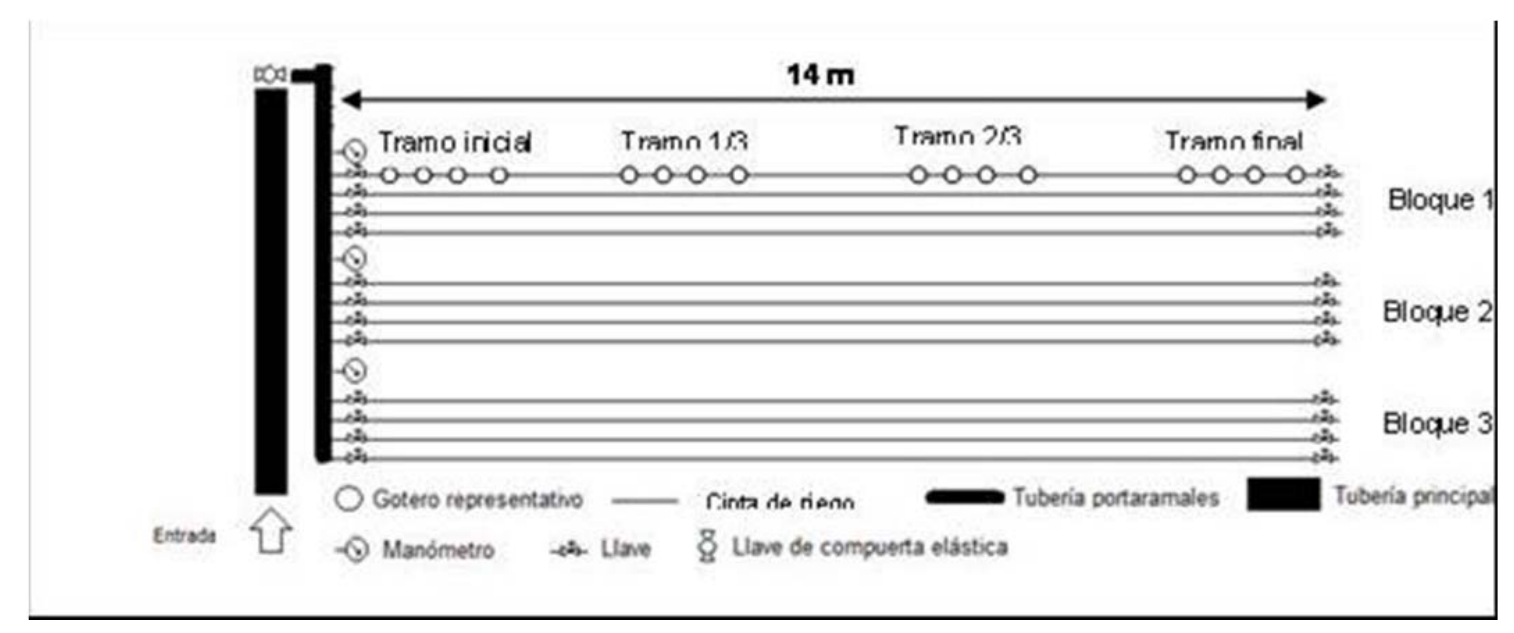

Figura 2. Diseño experimental del ensayo

Se ha empleado agua regenerada procedente de la Comunidad de Regantes Las Cuatro Vegas, concesionaria de las aguas residuales depuradas de la ciudad de Almería.

Se han realizado una serie de evaluaciones periódicas de cada unidad de riego, cuantificando el volumen de agua desalojado por cada emisor, y posteriormente se ha calculado el coeficiente de uniformidad del caudal (CUC) siguiendo el método clásico propuesto por Merrian y Keller (1978) (ecuación 3).

$$
\text { CUC }=\left(q_{25 \%} / q_{m}\right) \times 100
$$

(Ecuación 3)

Donde:

$\mathbf{q}_{25 \%}=$ caudal medio del $25 \%$ de emisores con menor descarga $\left(L h^{-1}\right)$.

$\mathbf{q}_{\mathbf{m}}=$ caudal medio de todos los emisores evaluados $\left(L h^{-1}\right)$.

Los valores de CUC fueron clasificados según by Merriam y Keller (1978) (Tabla 2).

Tabla 2. Clasificación del coeficiente de uniformidad de caudal (CUC) por Merriam y Keller (1978).

\begin{tabular}{|c|c|}
\hline CUC & Clasificación \\
\hline$>95 \%$ & Excelente \\
\hline $85 \%-95 \%$ & Bueno \\
\hline $80 \%-85 \%$ & Aceptable \\
\hline $70 \%-80 \%$ & Pobre \\
\hline$<70 \%$ & Inaceptable \\
\hline
\end{tabular}

\section{3- Resultados y Conclusiones}

Por lo que respecta al coeficiente de variación de fabricación, en diez de los modelos el material recibido es de categoría $A(C V<5)$. Dos modelos se clasifican como categoría $B$ $(5<\mathrm{CV}<10)$ y solo un modelo tiene un coeficiente de variación fuera de categoría (tabla 3$)$. El exponente de descarga de la fórmula de gasto oscila en los emisores turbulentos de laberinto entre 0,46 y 0,53 , en un rango similar a los emisores instalados en ramal convencional (tabla 3). 
Tabla 3. Resultados obtenidos en banco de ensayo

\begin{tabular}{|c|c|c|c|}
\hline Modelo & Fórmula de gasto * & CV & Categoría \\
\hline 1 & $\mathrm{Q}=1,24^{\star} \mathrm{H}^{0,49}$ & 4,07 & $\mathrm{~A}$ \\
\hline 2 & $\mathrm{Q}=0,78^{*} \mathrm{H}^{0,47}$ & 2,85 & $\mathrm{~A}$ \\
\hline 3 & $\mathrm{Q}=1,42^{\star} \mathrm{H}^{0,51}$ & 1,96 & $\mathrm{~A}$ \\
\hline 4 & $\mathrm{Q}=1,45^{\star} \mathrm{H}^{0,51}$ & 2,01 & $\mathrm{~A}$ \\
\hline 5 & $\mathrm{Q}=2,20^{\star} \mathrm{H}^{0,53}$ & 1,70 & $\mathrm{~A}$ \\
\hline 6 & $\mathrm{Q}=1,60^{\star} \mathrm{H}^{0,46}$ & 1,71 & $\mathrm{~A}$ \\
\hline 7 & $\mathrm{Q}=1,74^{*} \mathrm{H}^{-0,06}$ & 2,02 & $\mathrm{~A}$ \\
\hline 8 & $\mathrm{Q}=0,69^{\star} \mathrm{H}^{0,48}$ & 2,56 & $\mathrm{~A}$ \\
\hline 9 & $\mathrm{Q}=1,67^{\star} \mathrm{H}^{0,92}$ & 10,59 & $\mathrm{f} . \mathrm{C}$. \\
\hline 10 & $\mathrm{Q}=1,27^{*} \mathrm{H}^{0,51}$ & 5,16 & $\mathrm{~B}$ \\
\hline 10 & $\mathrm{Q}=0,96^{\star} \mathrm{H}^{0,48}$ & 5,21 & $\mathrm{~B}$ \\
\hline 11 & $\mathrm{Q}=0,85^{\star} \mathrm{H}^{0,48}$ & 2,04 & $\mathrm{~A}$ \\
\hline 12 & $\mathrm{Q}=1,15^{\star} \mathrm{H}^{0,46}$ & 2,04 & \\
\hline
\end{tabular}

En la evaluación de campo todos los emisores han presentado valores de uniformidad elevados al comienzo de la campaña de riego. Cinco de las cintas se clasifican como excelentes y el resto como buenas. Al finalizar las 114 horas de trabajo decae sustancialmente la uniformidad. Sólo una de las cintas presenta una calificación de excelente y 9 calificación de bueno. El resto quedan en el rango de aceptable ( 2 modelos) y pobre ( 1 modelo) (tabla 4, figuras 3 y 4). La calificación de aceptable ( $85>C U C>80)$ no se puede considerar apropiada cuando se riegan cultivos hortícolas de alto valor, por lo que sería recomendable la sustitución de las cintas cuando se alcancen dichos valores de uniformidad.

Tabla 4. Cálculo de los la media de las 3 repeticiones, de los coeficientes de uniformidad de caudal (CUC) inicial y tras $114 \mathrm{~h}$ de trabajo

\begin{tabular}{|l|c|c|c|c|}
\hline MODELO & $\begin{array}{c}\text { CUC } \\
\text { INICIAL }\end{array}$ & Calificación & $\begin{array}{c}\text { CUC } \\
\mathbf{1 1 4} \text { Horas }\end{array}$ & Calificación \\
\hline MODELO 1 & 92,68 & Bueno & 83,6 & Aceptable \\
\hline MODELO 2 & 90,68 & Bueno & 89,7 & Bueno \\
\hline MODELO 3 & 95,57 & Excelente & 91,4 & Bueno \\
\hline MODELO 4 & 96,33 & Excelente & 84,6 & Aceptable \\
\hline MODELO 5 & 96,72 & Excelente & 93,2 & Bueno \\
\hline MODELO 6 & 96,15 & Excelente & 95,8 & Excelente \\
\hline MODELO 7 & 96,39 & Excelente & 94,7 & Bueno \\
\hline MODELO 8 & 94,59 & Bueno & 87,5 & Bueno \\
\hline MODELO 9 & 94,12 & Bueno & 72,3 & Pobre \\
\hline MODELO 10 & 93,31 & Bueno & 87,8 & Bueno \\
\hline MODELO 11 & 91,23 & Bueno & 87,3 & Bueno \\
\hline MODELO 12 & 90,01 & Bueno & 94,5 & Bueno \\
\hline MODELO 13 & 94,96 & Bueno & 93,4 & Bueno \\
\hline
\end{tabular}

El comportamiento tras la finalización de la primera campaña de riego se puede considerar equiparable al obtenido en un estudio previo con una selección de 20 emisores en ramal convencional y este tipo de aguas. En este caso de un $85 \%$ de emisores con la calificación de excelente al inicio, sólo el 30\% la mantenía al finalizar la campaña de riego (Baeza et al. 2015). 


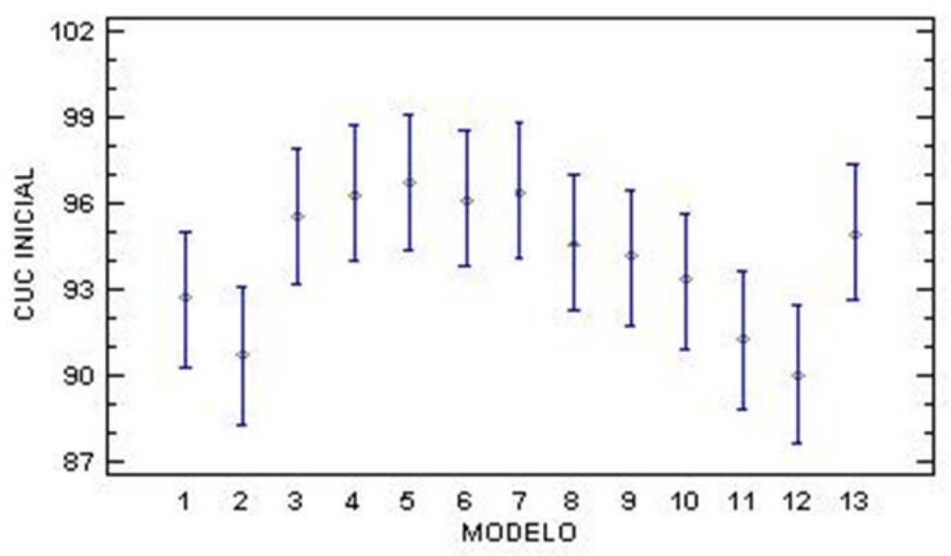

Figura 3. Coeficiente de Uniformidad de Caudal (CUC) inicial, expresado en tanto por ciento, de los 13 modelos analizados, con la representación del intervalo de Mínima Diferencia Significativa (LSD) al 95\%.

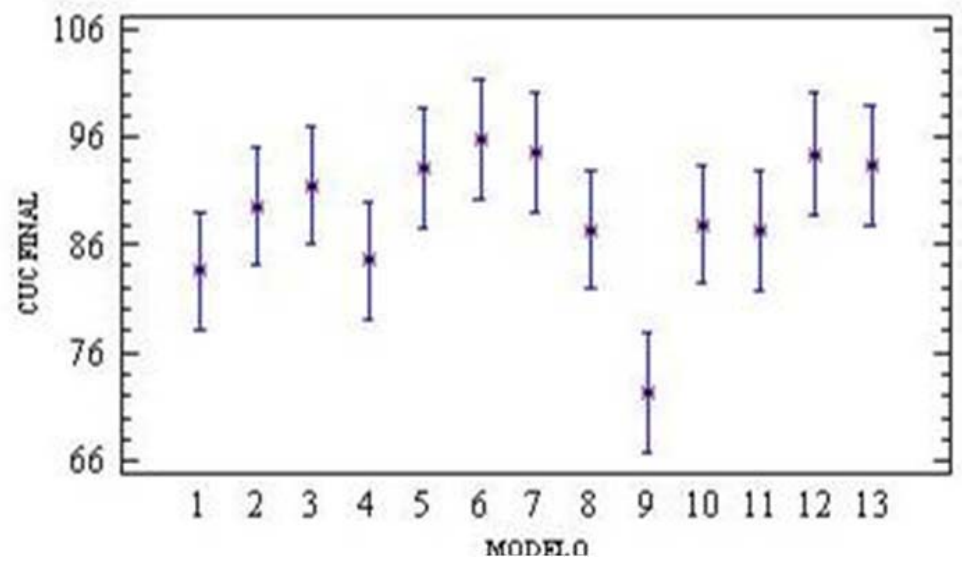

Figura 4. Coeficiente de Uniformidad de Caudal (CUC) final, expresado en tanto por ciento, de los 13 modelos analizados, con la representación del intervalo de Mínima Diferencia Significativa (LSD) al 95\%.

Tras analizar los resultados de laboratorio y campo, se puede concluir que las cintas de riego localizado pueden ser una alternativa a los ramales de riego convencionales cuando se riega con aguas residuales urbanas regeneradas, siempre que se elija un material de una mínima calidad y el ratio de sustitución garantice una uniformidad alta. Este estudio se va a ampliar con una segunda campaña de riego con el fin de analizar el comportamiento de las cintas a más largo plazo.

El coste de las cintas y goteros en tubería convencional es muy variable. Como referencia se puede considerar que el coste de la cinta de doble pared es un $50 \%$ del de la cinta con emisores integrados de pastilla, que a su vez suele corresponder con el $50 \%$ del ramal de riego en tubería convencional con emisores similares. La decisión de elegir uno u otro material requiere de un estudio económico previo. En cualquier caso la decisión tomada debe garantizar una uniformidad de riego alta y permanente.

\section{Bibliografía}

Agencia Andaluza del Agua. 2009. Posibilidad de reutilizar las aguas residuales regeneradas. Documento estratégico. 
Baeza, R., Contreras, J.I., Martín, F., Zapata, A. \& López, J.G. 2012. Estudio prospectivo de los emisores de riego localizado instalados en la zona regable del Bajo Andarax con aguas residuales urbanas regeneradas. XXX Congreso Nacional de Riegos. Albacete 12 al 14 de junio.

Baeza, R., Contreras, J.I., Trujillo, J. \& Alonso, F. 2015. Comportamiento de una selección de emisores para fertirriego de cultivos hortícolas en invernadero con aguas regeneradas. XXXIII Congreso Nacional de Riegos. Valencia 16 al 18 de junio.

Baeza, R., López, J.G., Domínguez, P., De Miguel, J. \& Cánovas, G. 2015. Jornada Técnica. Los recursos hídricos en el Campo de Dalías. Junta de Andalucía. Instituto de Investigación y Formación Agraria y Pesquera.

Baeza, R., Gavilán, P., Del Castillo, N., Berenguel, P. \& López, J.G. 2010. Programa de evaluación y asesoramiento en instalaciones de riego en invernadero con uso de dos fuentes distintas de agua: subterránea y regenerada. XXVIII Congreso Nacional de Riegos. León 15 al 17 de junio.

Fernández, M.D., Orgaz, F., Fereres, E., López, J.C., Céspedes, A., Pérez, J., Bonachela, S. \& Gallardo, M. 2001. Programación del riego de cultivos hortícolas bajo invernadero en el sudeste español. Estación Experimental Las Palmerillas. Cajamar

Junta de Andalucía. Consejería de Agricultura, Pesca y Medio Ambiente. 2014. Plan hidrológico de la Demarcación Hidrográfica de las Cuencas mediterráneas Andaluzas

Keller,J. \& Karmeli, D. 1974. Trickle irrigation design parameters. Transactions of de ASAE, 17(4): 678-684. 